\title{
Random Media and Eigenvalues of the Laplacian
}

\section{S. Ozawa}

Department of Mathematics, Faculty of Science, University of Tokyo, Hongo, Bunkyo-ku, Tokyo, 113, Japan

\begin{abstract}
Let $\beta$ be a fixed number $>1$. We remove $\left[\mathrm{m}^{\beta}\right]$-balls of centers $w_{1}, \ldots, w_{\left[m^{\beta}\right]}$ with the same radius $\alpha / m$ from a bounded domain $\Omega$ in $\mathbf{R}^{3}$. We consider the asymptotic behaviour of the $k^{\text {th }}$ eigenvalue of the Laplacian in $\Omega \backslash \overline{\left[m^{\beta} \text {-balls }\right]}$ under the Dirichlet condition as a random variable on a probability space $\left(w_{1}, \ldots, w_{\left[m^{\beta}\right]}\right) \in \Omega^{\left[m^{\beta}\right]}$, when $m \rightarrow \infty$.
\end{abstract}

\section{Introduction}

In the present note we consider a mathematical problem concerning random media. We consider a bounded domain $\Omega$ in $\mathbf{R}^{3}$ with smooth boundary $\Gamma$. We put

$$
B(\varepsilon ; w)=\left\{x \in \mathbf{R}^{3} ;|x-w|<\varepsilon\right\} .
$$

Fix $\beta \geqq 1$. Let $0<\mu_{1}(\varepsilon ; w(m)) \leqq \mu_{2}(\varepsilon ; w(m)) \leqq \ldots$ be the eigenvalues of $-\Delta(=-$ divgrad) in $\Omega_{\varepsilon, w(m)}=\Omega / \bigcup_{i=1}^{\widetilde{m}} B\left(\varepsilon ; w_{i}^{(m)}\right)$ under the Dirichlet condition on its boundary. Here $\tilde{m}$ denotes the largest integer which does not exceed $m^{\beta}$, and $w(m)$ denotes the set of $\tilde{m}$-points $\left\{w_{i}^{(m)}\right\}_{i=1}^{\tilde{m}} \in \Omega^{\tilde{m}}$. Let $V(x)>0$ be $C^{1}$-class function on $\bar{\Omega}$ satisfying

$$
\int_{\Omega} V(x) d x=1
$$

We consider $\Omega$ as the probability space with the probability density $V(x) d x$. Let $\Omega^{\tilde{m}}=\prod_{i=1}^{\tilde{m}} \Omega$ be the probability space with the product measure. The following result which is an elaboration of Kac's theorem (Kac [3]) was given in Ozawa [5].

Theorem A. Assume that $\beta=1$. Fix $\alpha>0$ and $k$. Then,

$$
\lim _{m \rightarrow \infty} \mathbb{P}\left(w(m) \in \Omega^{\tilde{m}} ; \quad m^{\tilde{\delta}}\left|\mu_{k}(\alpha / m ; w(m))-\mu_{k}^{V}\right|<\varepsilon\right)=1
$$

holds for any $\varepsilon>0$ and $\tilde{\delta} \in[0,1 / 4)$. Here $\mu_{k}^{V}$ denotes the $k^{\text {th }}$ eigenvalues of $-\Delta+4 \pi \alpha V(x)$ in $\Omega$ under the Dirichlet condition on $\Gamma$. 
In this paper we study the case $\beta>1$. In this case the sum of the radius of $\tilde{m}$-balls $B\left(\alpha / m ; w_{i}^{(m)}\right), i=1, \ldots, \tilde{m}$, tends to $\infty$ as $\tilde{m} \rightarrow \infty$. We see by the argument in RauchTaylor [9] that $\mu_{k}(\alpha / m ; w(m)) \rightarrow \infty$ if $\beta>1, V(x)>0$, and

$$
\lim _{m \rightarrow \infty} \tilde{m}^{-1} \sum_{i=1}^{\tilde{m}} f\left(w_{i}^{(m)}\right)=\int_{\Omega} f(x) V(x) d x
$$

for any fixed $f \in L^{\infty}(\Omega)$. We call the case $\beta>1, V(x)>0$ the soldifying case, following Rauch-Taylor.

The aim of this paper is to prove the following:

Theorem 1. Assume that $1 \leqq \beta<9 / 8$ and $V(x)>0$. Fix $\alpha>0$ and $k$. Then, there exists a constant $\delta(\beta)>0$ independent of $m$ such that

$$
\lim _{m \rightarrow \infty} \mathbb{P}\left(w(m) \in \Omega^{\tilde{m}} ; \quad m^{\delta^{\prime}-(\beta-1)}\left|\mu_{k}(\alpha / m ; w(m))-\mu_{k, m}^{V}\right|<\varepsilon\right)=1
$$

holds for any $\varepsilon>0$ and $\delta^{\prime} \in[0, \delta(\beta))$. Here $\mu_{k, m}^{V}$ denotes the $k^{\text {th }}$ eigenvalue of $-\Delta+4 \pi \alpha m^{\beta-1} V(x)$ in $\Omega$ under the Dirichlet condition on $\Gamma$.

Remark. There exist constants $C_{b}$ and $C_{u}$ such that

$$
C_{b}<m^{-(\beta-1)} \mu_{k, m}^{V}<C_{u} \text {. }
$$

Readers may refer to Papanicolaou and Varadhan [7, 8], Simon [10], Bensoussan et al. [1], Huruslov and Marchenko [2], Lions [4], Ozawa [5,6], and the literature cited there, for related topics.

\section{Probabilistic Consideration 1}

Fix $\beta \in(1,3)$. We consider the following condition $(\mathrm{D}-0)_{m}$ of $w(m) .(\mathrm{D}-0)_{m}$ : Assume that $\Omega \overline{\bigcup_{i=1}^{m} B\left(\alpha / m ; w_{i}^{(m)}\right)}$ is divided into the connected components $\omega_{1}(w(m)), \ldots, \omega_{g(w(m))}(w(m))$. Then, $g(w(m))=1$ or

$$
\max _{2 \leqq s \leqq g(w(m))} \operatorname{diam} \omega_{s(w(m))}(w(m)) \leqq m^{-1} \log m
$$

holds. Here diamz denotes the diameter of the set $\mathfrak{z}$.

We have the following:

Lemma 1. Assume that $\beta \in(1,3)$. Then,

$$
\lim _{m \rightarrow \infty} \mathbb{P}\left(w(m) \in \Omega^{\tilde{m}} ; w(m) \text { satisfies }(\mathrm{D}-0)_{m}\right)=1
$$

Proof. We suppose that $w(m)$ does not satisfy $(\mathrm{D}-0)_{m}$. Then, we see that there exists $[(\log m) / 2 \alpha]\left(=m_{*}\right)$ numbers $s_{1}(w(m)), \ldots, s_{m_{*}}(w(m))$, such that

$$
\operatorname{diam}\left(\bigcup_{k=1}^{m_{*}}\left\{w_{s_{k}(w(m))}^{(m)}\right\}\right\} \leqq 2 m^{-1} \log m .
$$


By a simple combinatorial argument we have

$$
\begin{aligned}
\mathbb{P}\left(w(m) \in \Omega^{\tilde{m}} ;(1.4) \text { holds }\right) & \leqq \tilde{m}^{m *} \mathbb{P}\left(\left(w_{1}^{(m)}, \ldots, w_{m_{*}}^{(m)}\right) \in \Omega^{m_{*}}\right. \\
\left|w_{j}^{(m)}-w_{k}^{(m)}\right| & \left.\leqq 2 m^{-1} \log m, j, k=1, \ldots, m_{*}\right) \\
& \leqq \tilde{m}^{m_{*}}\left(2 m^{-1}(\log m) C\right)^{3 m_{*}} \\
& \leqq\left(m^{\beta-3} / 8 C\right)^{m_{*}}(\log m)^{3 m_{*} \rightarrow 0}
\end{aligned}
$$

for $\beta \in(1,3)$. Thus, $(1.3)$ is proved.

We consider the following condition $(\mathrm{D}-\infty)_{m}$ of $w(m)$.

$(\mathrm{D}-\infty)_{m}$ : Take an arbitrary connected closed subset $\mathscr{R}_{m}$ of $\Gamma$ which contains the disk with radius (by the induced metric on $\Gamma$ ) $2 m^{-1} \log m$. Then,

$$
\mathscr{R}_{m} \sqrt{\bigcup_{i=1}^{\tilde{m}} B\left(\alpha / m ; w_{i}^{(m)}\right) \neq \phi} .
$$

It is easy to show that

$$
\lim _{m \rightarrow \infty} \mathbb{P}\left(w(m) \in \Omega^{\tilde{n}} ; \quad w(m) \text { satisfies }(\mathrm{D}-\infty)_{m}\right)=1 .
$$

\section{Idea of the Proof of Theorem 1}

We put $\gamma>\beta-1$. We abbreviate the largest positive number which does not exceed $m^{\gamma}$ as $m^{\prime}$. We put $m^{\prime \prime}=\left(m^{\prime}\right)^{1 / 2}$. Hereafter we always assume that $w(m)$ satisfies $(\mathrm{D}-0)_{m},(\mathrm{D}-\infty)_{m}$. We abbreviate $\omega_{1}(w(m))$ as $\omega$ for the sake of simplicity. Let $G_{\left(m^{\prime}\right)}(x, y ; w(m))$ be the Green's function of $\Delta-m^{\prime}$ in $\omega$ under the Dirichlet condition on its boundary satisfying

$$
\begin{gathered}
\left(\Delta_{x}-m^{\prime}\right) G_{\left(m^{\prime}\right)}(x, y ; w(m))=-\delta(x-y), \quad x, y \in \omega, \\
G_{\left(m^{\prime}\right)}(x, y ; w(m))=0, \quad x \in \partial \omega .
\end{gathered}
$$

Let $G_{\left(m^{\prime}\right)}(x, y)$ be the Green's function of $\Delta-m^{\prime}$ in $\Omega$ satisfying

$$
\begin{gathered}
\left(\Delta_{x}-m^{\prime}\right) G_{\left(m^{\prime}\right)}(x, y)=-\delta(x-y), \quad x, y \in \Omega, \\
G_{\left(m^{\prime}\right)}(x, y)=0, \quad x \in \Gamma .
\end{gathered}
$$

From now on we abbreviate $G_{\left(m^{\prime}\right)}(x, y)$ as $G(x, y)$. We introduce the following integral kernel function: We abbreviate $w_{i}^{(m)}$ as $w_{i}$ for the sake of simplicity.

$$
\begin{aligned}
h_{\left(m^{\prime}\right)}(x, y ; w(m))= & G(x, y)-(4 \pi \alpha / m) e^{m^{\prime \prime} \alpha / m} \sum_{i=1}^{\tilde{m}} G\left(x, w_{i}\right) G\left(w_{i}, y\right) \\
& +\sum_{s=2}^{m^{*}}(-4 \pi \alpha / m)^{s} e^{m^{\prime \prime} \alpha s / m} \\
& \cdot \sum_{(s)} G\left(x, w_{i_{1}}\right) G\left(w_{i_{1}}, w_{i_{2}}\right) \ldots G\left(w_{i_{s-1}}, w_{i_{s}}\right) G\left(w_{i_{s}}, y\right) .
\end{aligned}
$$

Here $m^{\prime \prime}=\left(m^{\prime}\right)^{1 / 2}$ and $m^{*}$ is a function of $m$ which is appropriately determined later. Here the indices $\left(i_{1}, \ldots, i_{s}\right)$ in $\sum_{(s)}$ run over all $1 \leqq i_{1}, \ldots, i_{s} \leqq \tilde{m}$ satisfying $i_{1} \neq i_{2}$, 
$i_{2} \neq i_{3}, \ldots, i_{s-1} \neq i_{s}$. An essential key to Theorem 1 is the fact that $h_{\left(m^{\prime}\right)}(x, y ; w(m))$, when we consider it as an integral kernel function on $\omega \times \omega$, is a nice approximation of $G_{\left(m^{\prime}\right)}(x, y ; w(m))$ in a rough sense, if $\beta-1$ is small. By a probabilistic consideration we view that $h_{\left(m^{\prime}\right)}(x, y ; w(m))$, when we consider it as an integral kernel function on $\Omega \times \Omega$, is a nice approximation of the integral kernel function of $\left(-\Delta+m^{\prime}+4 \pi \alpha m^{\beta-1} V(x)\right)^{-1}$ in a rough sense. Along this line we get Theorem 1.

\section{Preliminary Lemmas}

Lemma 2. Fix $\beta \in(1,3)$. Suppose that $u_{m} \in C^{\infty}(\omega)$ satisfies

$$
\begin{gathered}
\left(-\Delta+m^{\prime}\right) u_{m}(x)=0, \quad x \in \omega, \\
u_{m}(x)=0, \quad x \in \partial \omega \cap \Gamma,
\end{gathered}
$$

and

$$
\max \left\{\left|u_{m}(x)\right| ; x \in \partial B_{r} \cap \partial \omega\right\}=M_{r}(m), \quad r=1, \ldots, \tilde{m} .
$$

Here $B_{r}$ is an abbreviation of $B\left(\alpha / m ; w_{r}^{(m)}\right)$. If $\partial B_{r} \cap \partial \omega=\phi$, then we put $M_{r}(m)=0$. Under the above assumption, there exists a constant $C_{p}$ independent of such that

$$
\|u\|_{L^{p}(\omega)} \leqq C_{p} K_{p}(m) \sum_{r=1}^{\tilde{m}} M_{r}(m)
$$

holds, where

$$
K_{p}(m)= \begin{cases}m^{-(3 / p)}, & p>3, \\ m^{-1}\left(m^{\prime}\right)^{(-3+p) / 2 p}\left|\log \left(\left(m^{\prime}\right)^{1 / 2} / m\right)\right|^{1 / 3}, & p=3, \\ m^{-1}\left(m^{\prime}\right)^{(-3+p) / 2 p}, & 1 \leqq p<3\end{cases}
$$

Proof. By using the Hopf maximum principle we have

$$
|u(x)| \leqq C(\alpha / m) \sum_{r=1}^{\tilde{m}} \exp \left(-\left(m^{\prime}\right)^{1 / 2}\left|x-w_{r}\right|\right)\left|x-w_{r}\right|^{-1} M_{r}(m) .
$$

Notice that

$$
\left(\int_{\alpha / m}^{k} e^{-p m^{\prime \prime} t} t^{2-p} d t\right)^{1 / p} \leqq C_{p}^{\prime \prime} m^{(-3+p) / 2 p}\left(\int_{m^{\prime \prime} / m}^{m^{\prime \prime} K} t^{2-p} e^{-t} d t\right)^{1 / p}
$$

does not exceed $C_{p}^{\prime} K_{p}(m) m$. Thus, we get (4.1). q.e.d.

We have the following:

Lemma 3. Fix $\beta \in(1,3)$. Assume that $w(m)$ satisfies $(\mathrm{D}-0)_{m}$ and $(\mathrm{D}-\infty)_{m}$. Fix an arbitrary $\sigma \in(0,1]$. Then, there exists a constant $C_{(\sigma)}$ independent of $m$ such that

$$
\begin{aligned}
& \max _{x \in \partial B_{r} \cap \omega}\left|G\left(x, w_{i}\right)-G\left(w_{r}, w_{i}\right)\right| \leqq C_{(\sigma)}(\alpha / m)^{\sigma}\left|w_{i}-w_{r}\right|^{-1-\sigma} e^{-m^{\prime \prime}\left|w_{\imath}-w_{r}\right| / C_{(\sigma)}} \\
& \max _{x \in \partial B_{r} \cap \omega}\left|S\left(x, w_{r}\right) G\left(w_{r}, w_{i}\right)\right| \leqq C_{(\sigma)}(\alpha / m)^{\sigma}(\log m)\left|w_{i}-w_{r}\right|^{-1-\sigma} e^{-m^{\prime \prime}\left|w_{i}-w_{r}\right| / C_{(\sigma)}}
\end{aligned}
$$

hold. 
Fig. 1

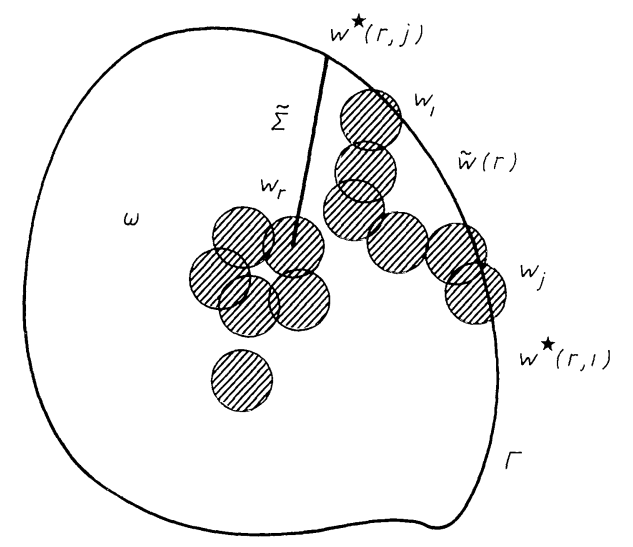

Proof. It should be remarked that $B_{r}$ and $B_{i}$ may have an intersection. We put

$$
\operatorname{Cone}\left(B_{r} \backslash B_{i}\right)=\left\{y=\theta w_{r}+(1-\theta) x ; x \in \partial B_{r} \backslash B_{i}, \theta \in[0,1]\right\} \text {. }
$$

By a simple geometrical observation we see that there exists a constant $C_{* *}$ independent of $w_{r}, w_{i}, m$ such that

$$
\left|w_{r}-w_{i}\right| \leqq C_{* *} \operatorname{dist}\left(\operatorname{Cone}\left(B_{r} \backslash B_{i}\right), w_{i}\right)
$$

holds. We see that the left side of (4.2) does not exceed

$$
C_{\sigma}^{\prime}(\alpha / m)^{\sigma}\left\|G\left(\cdot, w_{i}\right)\right\|_{C^{\sigma}\left(\operatorname{Cone}\left(B_{r} \backslash B_{i}\right)\right)} .
$$

Here $C^{\sigma}(F)$ denotes the usual Hölder space. Thus, we get (4.2).

We want to prove (4.3). Take $r$ such that $\partial B_{r} \cap \partial \omega \neq \phi$. Then, $\operatorname{dist}\left(w_{r}, \partial \omega \cap \Gamma\right)$ $\geqq(\alpha / m)$. By a simple geometrical observation including $(\mathrm{D}-\infty)_{m}$, we prove the following: Fix $r$ and $i$. Then, there exists a constant $\widetilde{E}>1$ independent of $m, r, i$ such that we can take $w^{*}(r, i) \in \partial \omega \cap \Gamma$ satisfying

$$
\operatorname{dist}\left(w_{r}, w^{*}(r, i)\right) \leqq \widetilde{E}(\log m) \operatorname{dist}\left(w_{r}, \Gamma \backslash \bar{B}_{r}\right),
$$

$\operatorname{dist}\left(w_{i}, \widetilde{\Sigma}\right) \geqq \tilde{E}^{-1}\left|w_{r}-w_{i}\right|$, where

$$
\tilde{\Sigma}=\bigcup_{0 \leqq}\left\{\theta w_{r}+(1-\theta) w^{*}(r, i)\right\} \quad \text { (see Fig. 1). }
$$

Take $\tilde{w}(r) \in \Gamma \backslash \bar{B}_{r}$ such that $\operatorname{dist}\left(w_{r}, \tilde{w}(r)\right)=\operatorname{dist}\left(w_{r}, \Gamma \backslash \bar{B}_{r}\right)$. Then,

$$
\begin{aligned}
\left|w_{r}-\tilde{w}(r)\right|^{-1} G\left(w_{r}, w_{i}\right) \leqq & \tilde{E}(\log m)\left|w_{r}-w^{*}(r, i)\right|^{-1}\left|G\left(w_{r}, w_{i}\right)-G\left(w^{*}(r, i), w_{i}\right)\right| \\
\leqq & \tilde{E}(\log m)(\alpha / m)^{\sigma-1}\left|w_{r}-w^{*}(r, i)\right|^{-\sigma} \\
& \cdot\left|G\left(w_{r}, w_{i}\right)-G\left(w^{*}(r, i), w_{i}\right)\right| .
\end{aligned}
$$

We see that

$$
\left\|G\left(\cdot, w_{i}\right)\right\|_{C^{\sigma}(\tilde{\Sigma})} \leqq C\left|w_{r}-w_{i}\right|^{-1-\sigma} \exp \left(-m^{\prime \prime}\left|w_{r}-w_{i}\right| / 2\right)
$$

holds. By a simple observation on the boundary behaviour of the Green's function we have

$$
\max _{x \in \partial B_{r} \cap \partial \omega}\left|S\left(x, w_{r}\right)\right| \operatorname{dist}\left(w_{r}, w(r)\right) \leqq C
$$


for a constant $C$ independent of $m, r$. In summing up these facts we get the desired result. q.e.d.

\section{Approximation of Green's Function}

Put

$$
\left(\mathbb{G}_{\left(m^{\prime}\right)} f\right)(x)=\int_{\omega} G_{\left(m^{\prime}\right)}(x, y ; w(m)) f(y) d y, \quad x \in \omega
$$

and

$$
\left(\mathbb{H}_{\left(m^{\prime}\right)} f\right)(x)=\int_{\omega} h_{\left(m^{\prime}\right)}(x, y ; w(m)) f(y) d y, \quad x \in \omega .
$$

Put $\mathbb{Q}_{\left(m^{\prime}\right)}=\mathbb{G}_{\left(m^{\prime}\right)}-\mathbb{H}_{\left(m^{\prime}\right)}$. Then, it satisfies

$$
\begin{gathered}
\left(-\Delta_{x}+m^{\prime}\right)\left(\mathbb{Q}_{\left(m^{\prime}\right)} f\right)(x)=0, \quad x \in \omega, \\
\left(\mathbb{Q}_{\left(m^{\prime}\right)} f\right)(x)=0, \quad x \in \partial \omega \cap \Gamma,
\end{gathered}
$$

for any $f \in C_{0}^{\infty}(\omega)$. We have to estimate $\sum_{r=1}^{\tilde{m}}\left|\mathbb{Q}_{\left(m^{\prime}\right)} f(x)\right|_{x \in \partial B_{r} \cap \partial \omega}$ to get a bound for $\left\|\mathbb{Q}_{\left(m^{\prime}\right)} f\right\|_{L^{q}(\omega)}$. We here introduce the following decomposition (5.1) of $\mathbb{H}_{\left(m^{\prime}\right)} f$. Fix $r$. We put

$$
\begin{aligned}
& \left(I_{r}^{s}\left(m^{\prime}\right) f\right)(x)=\Sigma_{(s)}^{\prime} G\left(x, w_{i_{1}}\right) G\left(w_{i_{1}}, w_{i_{2}}\right) \ldots G\left(w_{i_{s-1}}, w_{i_{s}}\right)\left(\mathbb{G}_{\left(m^{\prime}\right)} f\right)\left(w_{i_{s}}\right) \\
& \quad-(4 \pi \alpha / m) e^{m^{\prime \prime} \alpha / m} \sum_{(s)} G\left(x, w_{r}\right) G\left(w_{r}, w_{i_{1}}\right) \ldots G\left(w_{i_{s-1}}, w_{i_{s}}\right)\left(\mathbb{G}_{\left(m^{\prime}\right)} f\right)\left(w_{i_{s}}\right)
\end{aligned}
$$

for $s \geqq 1$. Here the indices in $\Sigma_{(s)}^{\prime}$ run over all $1 \leqq i_{1}, i_{2}, \ldots, i_{s} \leqq \tilde{m}$ such that $i_{1} \neq r$, $i_{2} \neq i_{1}, \ldots, i_{s} \neq i_{s-1}$. We put

$$
\left(I_{r}^{0}\left(m^{\prime}\right) f\right)(x)=\left(\mathbb{G}_{\left(m^{\prime}\right)} f\right)(x)-(4 \pi \alpha / m) e^{m^{\prime \prime} \alpha / m} G\left(x, w_{r}\right)\left(\mathbb{G}_{\left(m^{\prime}\right)} f\right)\left(w_{r}\right) .
$$

Then, it is easy to see that

$$
\begin{aligned}
\left(\mathbb{H}_{\left(m^{\prime}\right)} f\right)(x)= & \sum_{s=0}^{m^{*}}(-4 \pi \alpha / m)^{s} e^{m^{\prime \prime} \alpha s / m}\left(I_{r}^{s}\left(m^{\prime}\right) f\right)(x)+(-4 \pi \alpha / m)^{m^{*}} e^{m^{\prime \prime} \alpha m^{*} / m} \\
& \cdot \sum_{\left(m^{*}\right)}^{\prime} G\left(x, w_{i_{1}}\right) \ldots G\left(w_{i_{m^{*}-1}}, w_{i_{m^{*}}}\right)\left(\mathbb{G}_{\left(m^{\prime}\right)} f\right)\left(w_{i_{m^{*}}}\right) .
\end{aligned}
$$

We have

$$
\left.\left(I_{r}^{s}\left(m^{\prime}\right) f\right)(x)\right|_{x \in \partial B_{r} \cap \hat{\partial} \omega}=\left.\left(L_{r}^{s}\left(m^{\prime}\right) f\right)(x)\right|_{x \in \partial B_{r} \cap \partial \omega}+\left.\left(N_{r}^{s}\left(m^{\prime}\right) f\right)(x)\right|_{x \in \partial B_{r} \cap \hat{\partial} \omega}
$$

where

$$
\begin{aligned}
\left.\left(L_{r}^{S}\left(m^{\prime}\right) f\right)(x)\right|_{x \in \partial B_{r} \cap \hat{\partial} \omega}= & \Sigma_{(s)}^{\prime}\left(G\left(x, w_{i_{1}}\right)-G\left(w_{r}, w_{i_{1}}\right)\right) G\left(w_{i_{1}}, w_{i_{2}}\right) \\
& \ldots G\left(w_{i_{s-1}}, w_{i_{s}}\right)\left(\mathbb{G}_{\left(m^{\prime}\right)} f\right)\left(w_{i_{s}}\right)
\end{aligned}
$$

and

$$
\begin{aligned}
\left.\left(N_{r}^{s}\left(m^{\prime}\right) f\right)(x)\right|_{x \in \partial B_{r} \cap \partial \omega}= & (-4 \pi \alpha / m) e^{m \alpha / m} \sum_{(s)}^{\prime} S\left(x, w_{r}\right) G\left(w_{r}, w_{i_{1}}\right) \\
& \ldots G\left(w_{i_{s-1}}, w_{i_{s}}\right)\left(\mathbb{G}_{\left(m^{\prime}\right)} f\right)\left(w_{i_{s}}\right) .
\end{aligned}
$$


Here $S(x, y)=G(x, y)-(4 \pi|x-y|)^{-1} e^{-m^{\prime \prime}|x-y|}$. We know that $S(x, y) \in C^{\infty}(\Omega \times \Omega)$. By using the Hölder inequality we see that

$$
\begin{aligned}
\max _{x \in \partial B_{r} \cap \partial \omega}\left|I_{r}^{0}\left(m^{\prime}\right) f(x)\right| & \leqq C_{\theta}(\alpha / m)^{\theta}\left(\int_{0}^{k} e^{-m^{\prime \prime} r} r^{2-(1+\theta) p^{\prime}} d r\right)^{1 / p^{\prime}}\|f\|_{L^{p}(\omega)} \\
& \leqq C_{\theta}^{\prime} m^{-\theta-\left(3-(1+\theta) p^{\prime}\right) / 2 p^{\prime}}\|f\|_{L^{p}(\omega)}
\end{aligned}
$$

holds for $p^{\prime}<3 /(1+\theta)$. Here $p^{-1}+p^{-1}=1$. Hereafter we assume that $m^{\prime} m^{*} / m \mapsto 0$ as $m \mapsto \infty$. Observing Lemma 3, (5.2), (5.3), (5.4), we get the following:

$$
\sum_{r=1}^{\tilde{m}}\left|I_{r}^{S}\left(m^{\prime}\right) f(x)\right|_{\mid x \in \hat{\partial} B_{r} \cap \hat{\partial} \omega} \leqq C_{(\sigma)}(\alpha / m)^{\sigma}\left(m^{\prime}\right)^{\left(-3+p^{\prime}\right) / 2 p^{\prime}} \#_{(s)}^{(\sigma)}\|f\|_{L^{p}(\omega)},
$$

for $3 / 2<p<\infty, \sigma \in(0,1]$, where

$$
\#_{(s)}^{(\sigma)}=\sum_{(s+1)}\left|w_{i_{1}}-w_{i_{2}}\right|^{-1-\sigma} \exp \left(-m^{\prime \prime}\left|w_{i_{1}}-w_{i_{2}}\right|\right) G\left(w_{i_{2}}, w_{i_{3}}\right) \ldots G\left(w_{i_{s}}, w_{i s+1}\right) .
$$

In summing up these facts we get

Proposition 1. Fix $\beta \in(1,3)$. Assume that $w(m)$ satisfies $(\mathrm{D}-0)_{m}$ and $(\mathrm{D}-\infty)_{m}$. Assume that $m^{\prime} m^{*} / m \rightarrow 0$ as $m \rightarrow \infty$. Then,

$$
\left\|\mathbb{Q}_{\left(m^{\prime}\right)} f\right\|_{L^{q}(\omega)} \leqq C K_{q}(m) J\left(m, m^{*}, p, \sigma, \theta\right)\|f\|_{L^{p}(\omega)}
$$

holds for any $\sigma \in(0,1], \theta \in(0,1], p$ satisfying $3 /(2-\theta)<p \leqq \infty$. Here

$$
\begin{aligned}
J\left(m, m^{*}, p, \sigma, \theta\right)= & \tilde{m} m^{-\theta-\left(3-(1+\theta) p^{\prime}\right) / 2 p^{\prime}} \\
& +|\log m| m^{-\sigma}\left(m^{\prime}\right)^{\left(-3+p^{\prime}\right) / 2 p^{\prime}} \sum_{s=1}^{m^{*}}(4 \pi \alpha / m)^{s} \#_{(s)}^{(\sigma)} \\
& +(4 \pi \alpha / m)^{m^{*}}\left(m^{\prime}\right)^{\left(-3+p^{\prime}\right) / 2 p^{\prime}} \#_{\left(m^{*}\right)}^{(0)} .
\end{aligned}
$$

\section{Probabilistic Consideration 2}

It is easy to see that

$$
\begin{aligned}
\int_{\Omega} \ldots & \int_{\Omega} \frac{e^{-m^{\prime \prime}\left(\left|y_{1}-y_{2}\right|+\ldots+\left|r_{s}-r_{s+1}\right|\right) / K}}{\left|y_{1}-y_{2}\right|^{1+\sigma}\left|y_{2}-y_{3}\right| \ldots\left|y_{s}-y_{s-1}\right|\left|y_{s+1}-y_{s}\right|} \\
& \cdot d y_{1} d y_{2} \ldots d y_{s+1} \leqq C_{0}\left(C_{*} m^{\prime}\right)^{-s+(\sigma / 2)}
\end{aligned}
$$

holds for a constant $C_{0}, C_{*}$ independent of $m$. Thus,

$$
\mathbb{P}\left(w(m) \in \Omega^{\tilde{m}} ; \quad\left|m^{-s} \# \begin{array}{l}
(\sigma) \\
(s)
\end{array}\right|>\varepsilon\right) \leqq \varepsilon^{-1} C_{0}\left(C_{*} m^{\prime}\right)^{-s+(\sigma / 2)}\left(\tilde{m} m^{-1}\right)^{s} \tilde{m} .
$$

From now on we assume that $\gamma>\beta-1$. Then, $\tilde{m}\left(m^{\prime} m\right)^{-1}=m^{\beta-1-\gamma}$ tends to zero as $m \rightarrow \infty$. We have

$$
\begin{gathered}
\mathbb{P}\left(w(m) \in \Omega^{\tilde{m}} ;\right. \\
\left.\sup _{s}(8 \pi \alpha)^{s} m^{-s}\left(m^{\prime}\right)^{-(\sigma / 2)}\left|\#_{(s)}^{(\sigma)}\right|<\varepsilon\right) \geqq 1-\varepsilon^{-1} C_{0} \sum_{s=1}^{\tilde{m}}\left(C_{*} 8 \pi \alpha\left(m^{\prime}\right)^{-1} m^{-1} \tilde{m}\right)^{s} .
\end{gathered}
$$


We also have

$$
\begin{gathered}
P\left(w(m) \in \Omega^{\tilde{m}}\right. \\
\left.(8 \pi \alpha)^{m^{*}} m^{-m^{*}}\left(m^{\prime}\right)^{10}\left|\#_{\left(m^{*}\right)}^{(0)}\right|<\varepsilon\right) \geqq 1-\varepsilon^{-1}\left(C_{*} 8 \pi \alpha\left(m^{\prime} m\right)^{-1} \tilde{m}\right)^{m^{*}}\left(m^{\prime}\right)^{10} .
\end{gathered}
$$

In summing up these facts we get the following:

Proposition 2. Fix $\beta \in(1,3)$ and $\varepsilon>0$. Assume that $\gamma>\beta-1$ and $m^{\prime} m^{*} / m \rightarrow 0$ as $m \rightarrow \infty$. Fix $\sigma \in(0,1], \theta \in(0,1]$, $p$ satisfying $3 /(2-\theta)<p \leqq \infty$. Then,

$$
\lim _{m \rightarrow \infty} \mathbb{P}\left(w(m) \in \Omega^{\tilde{m}} ;(\mathrm{D}-0)_{m},(\mathrm{D}-\infty)_{m} \text { and }(6.1) \text { hold }\right)=1,
$$

where

$$
\left\|\mathbb{Q}_{\left(m^{\prime}\right)}\right\|_{L^{p}(\omega)} \leqq C H_{p}\left(m, m^{*}, p, \sigma, \theta, \varepsilon\right)
$$

Here

$$
\begin{aligned}
H_{q}\left(m, m^{*}, p, \sigma, \theta, \varepsilon\right)= & K_{q}(m)\left[\tilde{m} m^{-\theta-\left(3-(1+\theta) p^{\prime}\right) / 2 p^{\prime}}\right. \\
& +|\log m| m^{-\sigma}\left(m^{\prime}\right)^{\left(-3+p^{\prime}\right) / 2 p^{\prime}}\left(m^{\prime}\right)^{\sigma / 2} \tilde{m} \varepsilon \\
& \left.+\tilde{m}\left(m^{\prime}\right)^{-10} 2^{-m^{*}} \varepsilon\right]
\end{aligned}
$$

By the same argument as in Ozawa [5, Corollary 1] we can show the following Corollary 1 . Here we took $\theta=1, \sigma=1$, and $3<p$ as close as 3 . Hereafter we assume that $m^{*}=(\log m)^{2}$.

Corollary 1. Fix $\beta \in(1,3)$ and $\varepsilon>0$. Assume that $\gamma>\beta-1$. Fix an arbitrary $v>0$. Then,

$$
\lim _{m \rightarrow \infty} \mathbb{P}\left(w(m) \in \Omega^{\tilde{m}} ;(\mathrm{D}-0)_{m},(\mathrm{D}-\infty)_{m} \text { and }(6.2) \text { hold }\right)=1
$$

Here

$$
\left\|\mathbb{Q}_{\left(m^{\prime}\right)}\right\|_{L^{2}(\omega)} \leqq C\left[\tilde{m} m^{-2+v}\left(1+|\log m|\left(m^{\prime}\right)^{v} \varepsilon\right)\right] .
$$

We here consider the condition on $\beta, \gamma$ such that

$$
m^{\delta(\beta, \gamma)+2 \gamma-(\beta-1)}\left\|\mathbb{Q}_{\left(m^{\prime}\right)}\right\|_{L^{2}(\omega)}=o(1)
$$

holds for some $\delta(\beta, \gamma)>0$ as $m \rightarrow \infty$. Assume that $\gamma<1 / 2$. Then, there exists $v>0$, $\delta(\beta, \gamma)>0$ such that

[the right side of $(6.2)] \times m^{\delta(\beta, \gamma)+2 \gamma-(\beta-1)}$

tends to zero as $m \rightarrow \infty$. In summing up these results we have the following:

Proposition 3. Fix $0 \leqq \beta-1<\gamma<1 / 2, \varepsilon>0$. Then,

$$
\lim _{m \rightarrow \infty} \mathbb{P}\left(w(m) \in \Omega^{\tilde{m}} ;(\mathrm{D}-0)_{m},(\mathrm{D}-\infty)_{m} \text { and }(6.3) \text { hold }\right)=1 \text {. }
$$

Let $\widetilde{\mathbb{H}}_{\left(m^{\prime}\right)}$ be the integral operator defined by

$$
\left(\widetilde{\mathbb{H}}_{\left(m^{\prime}\right)} f\right)(x)=\int_{\Omega} h_{\left(m^{\prime}\right)}(x, y ; w(m)) d y, \quad x \in \Omega .
$$


Let $\chi_{\omega}$ (resp., $\tilde{\chi}_{\omega}$ ) be the characteristic function of $\omega$ (resp., $\left.\Omega \backslash \bar{\omega}\right)$. Fix $\psi \in L^{2}(\Omega)$. Put $g_{\psi}(m ; x)=\left(\widetilde{\mathbb{H}}_{\left(m^{\prime}\right)}\left(\tilde{\chi}_{\omega} \psi\right)\right)(x)$ for $x \in \omega$. We see that $\left(-\Delta+m^{\prime}\right) g_{\psi}(m ; x)=0, x \in \omega$, and $g_{\psi}(m ; x)=0$ for $x \in \partial \omega \cap \Gamma$. We want to estimate $\left\|g_{\psi}(m ; \cdot)\right\|_{L^{2}(\omega)}$ by using Lemma 2 . By a simple consideration on

$$
\sum_{r=1}^{\tilde{m}} \max \left\{\left|g_{\psi}(m ; x)\right| ; x \in \partial B_{r} \cap \partial \omega\right\}
$$

we know that

$$
\lim _{m \rightarrow \infty} \mathbb{P}\left(w(m) \in \Omega^{\tilde{m}} ;(\mathrm{D}-0)_{m},(\mathrm{D}-\infty)_{m} \text { and }(6.4) \text { hold }\right)=1 .
$$

Here (6.4) is the statement: there exists a constant $C_{(\theta)}$ independent of $m$ such that

$$
\left\|g_{\psi}(m ; \cdot)\right\|_{L^{2}(\omega)} \leqq C_{(\theta)} H_{2}\left(m, m^{*}, 2,1, \theta, \varepsilon\right)\left\|\tilde{\chi}_{\omega} \psi\right\|_{L^{2}(\Omega)}
$$

holds for any fixed $\theta \in(0,1 / 2)$.

Let $\tilde{\mu}_{j, m}$ be the $j^{\text {th }}$ eigenvalue of $-\Delta+m^{\prime}+4 \pi \alpha m^{\beta-1} V(x)$ in $\Omega$ under the Dirichlet condition on $\Gamma$. Let $\left\{\varphi_{j, m}\right\}_{j=1}^{\infty}$ be a complete orthonormal basis of eigenfunctions of $-\Delta+m^{\prime}+4 \pi \alpha m^{\beta-1} V(x)$ in $\Omega$ under the Dirichlet condition on $\Gamma$ associated with $\tilde{\mu}_{j, m}$. We know that

$$
\max _{x \in \bar{\Omega}}\left|\varphi_{j, m}(x)\right| \leqq C \tilde{\mu}_{j, m}\left(m^{\prime}\right)^{-1 / 4} \leqq C^{\prime}\left(m^{\prime}\right)^{3 / 4},
$$

using the property of the Green operator $\widetilde{\mathbb{G}}_{(m)}$ of $-\Delta+m^{\prime}+4 \pi \alpha m^{\beta-1} V(x)$ in $\Omega$ under the Dirichlet condition on $\Gamma$. Thus,

$$
\left\|\tilde{\chi}_{\omega} \varphi_{j, m}\right\|_{L^{2}(\Omega)} \leqq C^{\prime}\left(m^{\prime}\right)^{3 / 4} m^{(\beta-3) / 2},
$$

using (6.5).

We take $\theta<1 / 2$ as close as $1 / 2$. Then

$$
H_{2}\left(m, m^{*}, 2,1, \theta, \varepsilon\right) \leqq \tilde{m}\left(m^{\prime}\right)^{-1 / 4} m^{-(3 / 2)+v}+m^{-2}|\log m| \tilde{m} \varepsilon
$$

holds for any positive $v>0$. By an elementary calculation we have the following:

Proposition 4. Fix $\beta \in[1,9 / 8)$. Then, there exists $\gamma \in(4(\beta-1), 1 / 2), \kappa_{*}(\beta)>0$ such that

$$
\lim _{m \rightarrow \infty} \mathbb{P}_{m}\left((\mathrm{D}-0)_{m},(\mathrm{D}-\infty)_{m} \text { hold, } m^{\kappa_{*}(\beta)+2 \gamma-(\beta-1)}\left\|\tilde{\mathbb{H}}_{\left(m^{\prime}\right)}\left(\tilde{\chi}_{\omega} \varphi_{j, m}\right)\right\|_{L^{2}(\omega)} \leqq \varepsilon\right)=1
$$

holds for any $\varepsilon>0$. Sect. 7.

It should be remarked that $\gamma>4(\beta-1)$ is the restriction which will appear in

\section{Convergence of $\widetilde{\mathbb{H}}_{\left(m^{\prime}\right)}$}

We here consider the convergence $\tilde{\mathbb{H}}_{\left(m^{\prime}\right)} \rightarrow\left(-\Delta+m^{\prime}+4 \pi \alpha m^{\beta-1} V(x)\right)^{-1}$ in a probabilistic context. We modify the discussion in Ozawa [5, Sect. 3]. See also Sect. 9 in this note. 
We examine the following term. Fix $u, v \in L^{2}(\Omega)$.

$$
\begin{aligned}
\mathscr{P}_{(s)}(u, v ; w(m))= & \tilde{m}^{-s} \sum_{(s)}\left(\mathbb{G}_{\left(m^{\prime}\right)} v\right)\left(w_{i_{1}}\right) G\left(w_{i_{1}}, w_{i_{2}}\right) \ldots G\left(w_{i_{s-1}}, w_{i_{s}}\right)\left(\mathbb{G}_{\left(m^{\prime}\right)} u\right)\left(w_{i_{s}}\right) \\
& -\int_{\Omega}\left(\mathbb{G}_{\left(m^{\prime}\right)}\left(V \mathbb{G}_{\left(m^{\prime}\right)}\right) s^{s} u\right)(x) v(x) d x \\
= & \sum_{h=1}^{s} J_{s, h}(u, v ; w(m)) .
\end{aligned}
$$

Here $\sum_{(1)} \ldots$ means

$$
\sum_{i=1}^{\tilde{m}}\left(\mathbb{G}_{\left(m^{\prime}\right)} v\right)\left(w_{i}\right)\left(\mathbb{G}_{\left(m^{\prime}\right)} u\right)\left(w_{i}\right)
$$

and where

$$
\begin{aligned}
& J_{s, s}(u, v ; w(m))=\tilde{m}^{-1} \sum_{i=1}^{\tilde{m}}\left(\mathbb{G}_{\left(m^{\prime}\right)} v\right)\left(w_{i}\right)\left(\mathbb{G}_{\left(m^{\prime}\right)}\left(V \mathbb{G}_{\left(m^{\prime}\right)}\right)^{s-1} u\right)\left(w_{i}\right) \\
& -\int_{\Omega}\left(\mathbb{G}_{\left(m^{\prime}\right)}\left(V \mathbb{G}_{\left(m^{\prime}\right)}\right)^{s} u\right)(x) v(x) d x, \\
& J_{s, s-1}(u, v ; w(m))=\tilde{m}^{-1} \sum_{i=1}^{\tilde{m}}\left(\mathbb{G}_{\left(m^{\prime}\right)} v\right)\left(w_{i}\right) \\
& \cdot\left\{\tilde{m}^{-1} \sum_{\substack{i_{2}=1 \\
i_{2} \neq i_{1}}}^{\tilde{m}} G\left(w_{i_{1}}, w_{i_{2}}\right)\left(\mathbb{G}_{\left(m^{\prime}\right)}\left(V \mathbb{G}_{\left(m^{\prime}\right)}\right)^{s-2} u\right)\left(w_{i_{2}}\right)\right. \\
& \left.-\left(\mathbb{G}_{\left(m^{\prime}\right)}\left(V \mathbb{G}_{\left(m^{\prime}\right)}\right)^{s-1} u\right)\left(w_{i_{1}}\right)\right\}, \\
& J_{s, s-q}(u, v ; w(m))=\tilde{m}^{-1} \sum_{i_{1}=1}^{\tilde{m}}\left(\mathbb{G}_{\left(m^{\prime}\right)} v\right)\left(w_{i_{1}}\right) \\
& \cdot\left(\tilde{m}^{-1} \sum_{\substack{i_{2}=1 \\
i_{2} \neq i_{1}}}^{\tilde{m}} G\left(w_{i_{1}}, w_{i_{2}}\right) \ldots \tilde{m}^{-1} \sum_{\substack{i_{q}=1 \\
i_{q} \neq i_{q}-1}}^{\tilde{m}} G\left(w_{i_{q-1}}, w_{i_{q}}\right)\right. \\
& \left\{\tilde{m}^{-1} \sum_{\substack{i_{q+1}=1 \\
i_{q+1} \neq i_{q}}}^{\tilde{m}} G\left(w_{i_{q}}, w_{i_{q+1}}\right)\left(\mathbb{G}_{\left(m^{\prime}\right)}\left(V \mathbb{G}_{\left(m^{\prime}\right)}\right)^{s-q-1} u\right)\left(w_{i_{q+1}}\right)\right. \\
& \left.-\left(\mathbb{G}_{\left(m^{\prime}\right)}\left(V \mathbb{G}_{\left(m^{\prime}\right)}\right)^{s-q} u\right)\left(w_{i_{q}}\right),(2 \leqq q \leqq s-1)\right\} \text {. }
\end{aligned}
$$

Put $\widetilde{P}_{s}(u, v ; w(m))=\mathscr{P}_{s}(u, v ; w(m))-J_{s, s}(u, v ; w(m))$. Let $\left\{u_{j}\right\}_{j=1}^{\infty}$ be a sequence such that $\left\|u_{j}\right\|_{L^{2}(\Omega)} \leqq 1$. The following inequality is easy to see:

$$
\begin{aligned}
& \left\|\left(\tilde{\mathbb{H}}_{\left(m^{\prime}\right)}-\widetilde{\mathbb{G}}_{(m)}\right) u_{m}\right\|_{L^{2}(\Omega)} \\
& \leqq \sum_{s=1}^{m^{*}}\left(4 \pi \alpha \tilde{m} m^{-1}\right)^{s}\left(\sup _{\|v\|_{L^{2}(\Omega)} \leqq} \leqq\left|\widetilde{P}_{s}\left(u_{m} \cdot v ; w(m)\right)\right|+K_{s}\left(u_{m} ; w(m)\right)\right) \\
& \quad+\sum_{s=m^{*}}^{\infty}\left(4 \pi \alpha \tilde{m} m^{-1}\right)^{s}\left\|\mathbb{G}_{\left(m^{\prime}\right)}\left(V \mathbb{G}_{\left(m^{\prime}\right)}\right)^{s} u_{m}\right\|_{L^{2}(\Omega)},
\end{aligned}
$$


where

$$
\begin{aligned}
K_{s}\left(u_{m} ; w(m)\right)= & \| \tilde{m}^{-1} \sum_{i=1}^{\tilde{m}} G\left(\cdot, w_{i}\right)\left(\mathbb{G}_{\left(m^{\prime}\right)}\left(V \mathbb{G}_{\left(m^{\prime}\right)}\right)^{s-1} u_{m}\right)\left(w_{i}\right) \\
& -\mathbb{G}_{\left(m^{\prime}\right)}\left(V \mathbb{G}_{\left(m^{\prime}\right)}\right) u^{s} u_{m}(\cdot) \|_{L^{2}(\Omega)} .
\end{aligned}
$$

Firstly we study the term in (7.1) which includes $\widetilde{\mathscr{P}}_{s}$. By using the Schwarz inequality as in Ozawa [5, Lemma 3], we have

$$
\begin{aligned}
\left|\widetilde{P}_{s}\left(u_{m}, v ; w(m)\right)\right| \leqq & \left\{\tilde{m}^{-1} \sum_{i=1}^{\tilde{m}}\left(\mathbb{G}_{\left(m^{\prime}\right)} v\right)\left(w_{i}\right)^{2}\right\}^{1 / 2} \\
& \cdot \sum_{q=1}^{s-1}\left(\tilde{m}^{-1} \tau(w(m))\right)^{q-1} \pi_{s-q}(u ; w(m))
\end{aligned}
$$

for $s \geqq 2$, where

$$
\begin{gathered}
\tau(w(m))=\left(\sum_{\substack{i, j=1 \\
i \neq j}}^{\tilde{m}} G\left(w_{i}, w_{j}\right)^{2}\right)^{1 / 2} \\
\pi_{s-q}(u ; w(m))=\left[\tilde { m } ^ { - 1 } \sum _ { i = 1 } ^ { \tilde { m } } \left\{\tilde{m}^{-1} \sum_{\substack{j=1 \\
j \neq i}}^{\tilde{m}} G\left(w_{i}, w_{j}\right)\left(\mathbb{G}_{\left(m^{\prime}\right)}\left(V \mathbb{G}_{\left(m^{\prime}\right)}\right)^{s-q-1} u\right)\left(w_{j}\right)\right.\right. \\
\left.\left.-\left(\mathbb{G}_{\left(m^{\prime}\right)}\left(V \mathbb{G}_{\left(m^{\prime}\right)}\right)^{s-q} u\right)\left(w_{i}\right)\right\}^{2}\right]^{1 / 2} .
\end{gathered}
$$

We have

$$
\left\{\tilde{m}^{-1} \sum_{i=1}^{\tilde{m}}\left(\mathbb{G}_{\left(m^{\prime}\right)} v\right)\left(w_{i}\right)^{2}\right\}^{1 / 2} \leqq C\left(m^{\prime}\right)^{-1 / 4}\|v\|_{L^{2}(\Omega)} .
$$

We want to show the following:

Lemma 4. Assume that $\gamma>4(\beta-1) \geqq 0$. Let $\left\{u_{j}\right\}_{j=1}^{\infty}$ be as before. Fix $\varepsilon>0$, $\zeta \in[0,1 / 4)$. Then,

$$
\lim _{m \rightarrow \infty} \mathbb{P}_{m}\left((\tilde{m})^{1 / 4}\left(m^{\top}\right)^{\zeta} \sum_{s=1}^{m^{*}}\left(4 \pi \alpha \tilde{m} m^{-1}\right)^{s} \sup _{\|v\|_{I^{2}(\Omega)}\left(\tilde{P}_{1}\right.}\left|\tilde{\mathscr{P}}_{s}\left(u_{m}, v ; w(m)\right)\right| \leqq \varepsilon\right)=1,
$$

where $\mathbb{P}_{m}(\cdot)$ denotes the probability $\mathbb{P}\left(w(m) \in \Omega^{\tilde{m}} ; \cdot\right)$.

We need some lemmas to get Lemma 4 . We have

Lemma 5. Let $\varrho$ be a fixed constant in $[0,1 / 4)$. Fix $\varepsilon>0$. Then,

$$
\lim _{m \rightarrow \infty} \mathbb{P}_{m}\left(\left(m^{\prime}\right)^{\tilde{Q}}\left|\tilde{m}^{-1} \tau(w(m))\right| \leqq \varepsilon\right)=1 .
$$

Proof. We have $\mathbb{E}\left(\tilde{m}^{-2} \tau(w(m))^{2}\right) \leqq C_{0}\left(m^{\prime}\right)^{-1 / 2}$. Here $\mathbb{E}(\cdot)$ denotes the expectation. Thus, (7.2) does not exceed $1-\varepsilon^{-2} C_{0}^{1 / 2}\left(m^{\prime}\right)^{2 \tilde{\varrho}-(1 / 2)}$. q.e.d. 
Lemma 6. Fix an arbitrary family of continuous functions on $\bar{\Omega}$ satisfying

$$
\max _{x \in \bar{\Omega}}\left|f_{h, m}(x)\right| \leqq D^{*}\left(C_{*} m^{\prime}\right)^{-h}
$$

for some constant $C_{*}>0$ and $D^{*}<\infty$. Put

$$
\begin{aligned}
\tilde{q}_{h, m}(w(m))= & \left(( C _ { * } m ^ { \prime } ) ^ { h / 2 } \left(\tilde { m } ^ { - 1 } \sum _ { i = 1 } ^ { m } \left\{\tilde{m}^{-1} \sum_{\substack{j=1 \\
j \neq i}}^{m} G\left(w_{i}, w_{j}\right) f_{h, m}\left(w_{j}\right)\right.\right.\right. \\
& \left.\left.\left.-\left(\mathbb{G}_{\left(m^{\prime}\right)} V f_{h, m}\right)\left(w_{i}\right)\right\}^{2}\right)\right) .
\end{aligned}
$$

Fix $\tilde{\mu} \in[0,2), \varepsilon>0$. Then

$$
\lim _{m \rightarrow \infty} \mathbb{P}_{m}\left((\tilde{m})^{1 / 2}\left(m^{\prime}\right)^{\tilde{\mu}} \sup _{h}\left|\tilde{q}_{h, m}\right| \leqq \varepsilon\right)=1
$$

Proof. We divide $\tilde{q}_{h, m}$ into three parts $\left(C_{*} m^{\prime}\right)^{h / 2}\left(L_{h, m}^{1}+L_{h, m}^{2}+L_{h, m}^{3}\right)$, where

$$
\begin{gathered}
L_{h, m}^{1}=\tilde{m}^{-1} \sum_{i=1}^{\tilde{m}}\left\{\tilde{m}^{-1} \sum_{\substack{j=1 \\
j \neq i}}^{\tilde{m}} G\left(w_{i}, w_{j}\right) f_{h, m}\left(w_{j}\right)\right\}^{2}, \\
L_{h, m}^{2}=-2 \tilde{m}^{-2} \sum_{\substack{i, j=1 \\
i \neq j}}^{\tilde{m}} G\left(w_{i}, w_{j}\right)\left(\mathbb{G}_{\left(m^{\prime}\right)} V f_{h, m}\right)\left(w_{i}\right) f_{h, m}\left(w_{j}\right), \\
L_{h, m}^{3}=\tilde{m}^{-1} \sum_{i=1}^{\tilde{m}}\left(\mathbb{G}_{\left(m^{\prime}\right)} V f_{h, m}\right)\left(w_{i}\right)^{2} .
\end{gathered}
$$

We put

$$
\left\langle L_{h, m}^{3}\right\rangle=\int_{\Omega}\left(\mathbb{G}_{\left(m^{\prime}\right)} V f_{h, m}\right)^{2}(x) d x
$$

It is easy to see that

$$
\mathbb{P}_{m}\left(\left|L_{h, m}^{3}-\left\langle L_{h, m}^{3}\right\rangle\right| \leqq \varepsilon\right) \leqq 4 \varepsilon^{-2} \tilde{m}^{-1} \widetilde{C}\left(C_{*} m^{\prime}\right)^{-4 h}\left(m^{\prime}\right)^{-4} .
$$

Therefore,

$$
\begin{aligned}
& \mathbb{P}_{m}\left((\tilde{m})^{1 / 2}\left(m^{\prime}\right)^{2+v} \sup _{h}\left(C_{*} m^{\prime}\right)^{h / 2}\left|L_{h, m}^{3}-\left\langle L_{h, m}^{3}\right\rangle\right| \leqq \varepsilon\right) \\
& \geqq 1-4 \varepsilon^{-2} \widetilde{C}\left(m^{\prime}\right)^{2 v} \sum_{h=1}^{\infty}\left(C_{*} m^{\prime}\right)^{-3 h} .
\end{aligned}
$$

We here review some elementary facts in probability theory. Let $g(x, y)$ be a square integrable function on $\Omega^{2}$. We have

$$
\begin{aligned}
& \mathbb{E}\left(\left\{(\tilde{m}(\tilde{m}-1))^{-1} \sum_{\substack{i, j=1 \\
i \neq j}}^{\tilde{m}}\left(g\left(w_{i}, w_{j}\right)-\mathbb{E}(g)\right)\right\}^{2}\right) \\
& \leqq(\tilde{m}(\tilde{m}-1))^{-2}\left\{\mathbb{E}\left(\sum_{\substack{i, j=1 \\
i \neq j}}^{\tilde{m}}\left(g\left(w_{i}, w_{j}\right)-\mathbb{E}(g)\right)^{2}\right)\right. \\
& \left.+4 \mathbb{E}\left(\sum_{\substack{i, j, h=1 \\
i \neq j, j \neq h, h \neq i}}^{\tilde{m}}\left|g\left(w_{i}, w_{j}\right)-\mathbb{E}(g)\right|\left|g\left(w_{i}, w_{h}\right)-\mathbb{E}(g)\right|\right)\right\} \text {. }
\end{aligned}
$$


The second term in the right side of the above does not exceed

$$
4 \tilde{m} \mathbb{E}\left(\sum_{\substack{i, j=1 \\ i \neq j}}^{\tilde{m}}\left(g\left(w_{i}, w_{j}\right)-E(g)\right)^{2}\right) .
$$

Thus, the term in the left side of $(7.7)$ does not exceed $(\tilde{m}-1)^{-1} 5\left(\mathbb{E}\left(g^{2}\right)\right.$ $\left.+3 \mathbb{E}(|g|)^{2}\right)$. Put

$$
g(x, y)=G(x, y)\left(\mathbb{G}_{\left(m^{\prime}\right)} V f_{h, m}\right)(x) f_{h, m}(y)
$$

Then, we have

$$
\|g\|_{L^{2}\left(\Omega^{2}\right)}^{2} \leqq C^{\prime \prime}\left(m^{\prime}\right)^{-5 / 2}\left(C_{*} m^{\prime}\right)^{-4 h} .
$$

Notice that $\mathbb{E}\left(L_{h, m}^{2}\right)=-2\left\langle L_{h, m}^{3}\right\rangle$. In summing up these facts we get

$$
\begin{aligned}
& \mathbb{P}_{m}\left(\tilde{m}^{(1 / 2)}\left(m^{\prime}\right)^{(5 / 4)+v^{\prime}} \sup _{h}\left(C_{*} m^{\prime}\right)^{h / 2}\left|L_{h, m}^{2}+2\left\langle L_{h, m}^{3}\right\rangle\right| \leqq \varepsilon\right) \\
& \geqq 1-6 \hat{C} \varepsilon^{-2}\left(m^{\prime}\right)^{2 v^{\prime}} \sum_{h=1}^{\infty}\left(C_{*} m^{\prime}\right)^{-3 h} .
\end{aligned}
$$

We want to examine $L_{h, m}^{1}$. We have $L_{h, m}^{1}=L_{h, m}^{1,1}+L_{h, m}^{1,2}$, where

$$
\begin{gathered}
L_{h, m}^{1,1}=\tilde{m}^{-3} \sum_{\substack{i, j, k=1 \\
i \neq j, j \neq k, k \neq i}}^{\tilde{m}} G\left(w_{i}, w_{j}\right) G\left(w_{i}, w_{k}\right) f_{h, m}\left(w_{j}\right) f_{h, m}\left(w_{k}\right) \\
L_{h, m}^{1,2}=\hat{m}^{-3} \sum_{\substack{i, j=1 \\
i \neq j}}^{\tilde{m}} G\left(w_{i}, w_{j}\right)^{2} f_{h, m}\left(w_{j}\right)^{2} .
\end{gathered}
$$

Let $\tilde{g}(x, y, z)$ be a square integrable function on $\Omega^{3}$. Then, we see that

$$
\mathbb{E}\left(\left\{\tilde{m}^{-3} \sum_{\substack{i, j, k=1 \\ i \neq j, j \neq k, k \neq i}}^{\tilde{m}} \tilde{g}\left(w_{i}, w_{j}, w_{k}\right)-E(\tilde{g})\right\}^{2}\right) \leqq C_{0} \tilde{m}^{-1}\left(\|\tilde{g}\|_{L^{2}\left(\Omega^{3}\right)}^{2}+\|\tilde{g}\|_{L^{1}\left(\Omega^{3}\right)}^{2}\right)
$$

holds for a constant $C_{0}$. We put $\tilde{g}(x, y, z)=G(x, y) G(x, z) f_{h, m}(y) f_{h, m}(z)$. Then, we have

$$
\|\tilde{g}\|_{L^{2}\left(\Omega^{3}\right)}^{2} \leqq C^{\prime}\left(m^{\prime}\right)^{-1}\left(C_{*} m^{\prime}\right)^{-4 h} .
$$

Therefore,

$$
\begin{aligned}
& \mathbb{P}_{m}\left(\tilde{m}^{(1 / 2)}\left(m^{\prime}\right)^{(1 / 2)+v^{\prime \prime}} \sup _{h}\left(C_{*} m^{\prime}\right)^{h / 2}\left|L_{h, m}^{1,1}-L_{h, m}^{3}\right| \leqq \varepsilon\right) \\
& \geqq 1-C_{0} \varepsilon^{-2}\left(m^{\prime}\right)^{2 v^{\prime \prime}} \sum_{h=1}^{\infty}\left(C_{*} m^{\prime}\right)^{-3 h} .
\end{aligned}
$$

We also have

$$
\begin{aligned}
& \mathbb{P}_{m}\left(\left(m^{\prime}\right)^{(1 / 2)+\tilde{v}}(\tilde{m})^{\tilde{\sigma}} \sup _{h}\left(C_{*} m^{\prime}\right)^{h / 2}\left|L_{h, m}^{1,2}\right| \leqq \varepsilon\right) \\
& \geqq 1-C_{0} \varepsilon^{-1} \tilde{m}^{\tilde{\sigma}-1}\left(m^{\prime}\right)^{\tilde{v}} \sum_{h=1}^{\infty}\left(C_{*} m^{\prime}\right)^{-3 h / 2} .
\end{aligned}
$$

By (7.6), (7.8), (7.9), (7.10), we have the following: 
Lemma 7. Fix an arbitrary sequence $\left\{u_{j}\right\}_{j=1}^{\infty}$ satisfying $\left\|u_{j}\right\|_{L^{2}(\Omega)} \leqq 1$. Then, there exists a constant $C$ such that

$$
\lim _{m \rightarrow \infty} \mathbb{P}_{m}\left((\tilde{m})^{1 / 4}\left(m^{\prime}\right)^{\varrho} \sup _{s}\left(C_{*} m^{\prime}\right)^{s / 4}\left|\pi_{s}\left(u_{m} ; w(m)\right)\right| \leqq \varepsilon\right)=1
$$

holds for any fixed $\varrho \in[0,1 / 4), \varepsilon>0$.

Proof. Put $f_{h, m}=\left(m^{\prime}\right)^{-3 / 4} \mathbb{G}_{\left(m^{\prime}\right)}\left(V G_{\left(m^{\prime}\right)}\right)^{h-1} u_{m}$. Then, it satisfies (7.3). We have $\left(C_{*} m^{\prime}\right)^{h / 2} \pi_{h}^{2}=\tilde{q}_{h, m}$. q.e.d.

Now the proof of Lemma 4 is easy. It reduces to the problem of convergence of

$$
\sum_{s=1}^{\infty}\left(4 \pi \alpha \tilde{m} m^{-1}\left(m^{\prime}\right)^{-\tilde{\varrho}}\right)^{s} .
$$

Here $\varrho$ is a fixed number in Lemma 5. If $\gamma>4(\beta-1)$, then we can take $\tilde{\varrho} \in[0,1 / 4)$ such that $m^{\beta-1-\tilde{\varrho} \gamma}=o\left(m^{-\varrho^{\prime \prime}}\right)$ for some $\varrho^{\prime \prime}>0$. q.e.d.

From now on we begin to study the term in (7.1) which includes $K_{s}$. Put $G^{(2)}(x, y)=\int_{\Omega} G(x, z) G(z, y) d z$. Then, $K_{s}\left(u_{m} ; w(m)\right)^{2}$ is equal to

$$
\begin{aligned}
& \left(\tilde{m}^{-2} \sum_{i, j=1}^{\tilde{m}} G\left(w_{i}, w_{j}\right)\left(\mathbb{G}_{\left(m^{\prime}\right)} u_{m}^{(s-1)}\right)\left(w_{i}\right)\left(\mathbb{G}_{\left(m^{\prime}\right)} u_{m}^{(s-1)}\right)\left(w_{j}\right)\right. \\
& \left.-\int_{\Omega}\left(\mathbb{G}_{\left(m^{\prime}\right)} u_{m}^{(s)}\right)(x)^{2} d x\right) \\
& -\left(2 \tilde{m}^{-1} \sum_{i=1}^{\tilde{m}}\left(\mathbb{G}_{\left(m^{\prime}\right)}^{2} u_{m}^{(s)}\right)\left(w_{i}\right)\left(\mathbb{G}_{\left(m^{\prime}\right)} u_{m}^{(s-1)}\right)\left(w_{i}\right)\right. \\
& \left.-2 \int_{\Omega}\left(\mathbb{G}_{\left(m^{\prime}\right)} u_{m}^{(s)}\right)(x)^{2} d x\right) .
\end{aligned}
$$

Here $u_{m}^{(s)}(x)$ denotes $\left(V \mathbb{G}_{\left(m^{\prime}\right)}\right)^{s} u_{m}(x)$. As we discussed before, we have

$$
\mathbb{E}\left(K_{s}\left(u_{m} ; w(m)\right)^{4}\right) \leqq \tilde{C}\left(I_{1}(m)+I_{2}(m)\right),
$$

where

$$
\begin{aligned}
I_{1}(m) & =\left|\left(\mathbb{G}_{\left(m^{\prime}\right)}^{2} u_{m}^{(s)}\right)\left(\mathbb{G}_{\left(m^{\prime}\right)} u_{m}^{(s-1)}\right)\right|_{C^{0}(\Omega)}^{2} \leqq C^{\prime \prime}\left(C^{\prime} m^{\prime}\right)^{-4 s-1}, \\
I_{2}(m) & =\iint_{\Omega \times \Omega}\left(G^{(2)}(x, y)\left(\mathbb{G}_{\left(m^{\prime}\right)} u_{m}^{(s-1)}\right)(x)\left(\mathbb{G}_{\left(m^{\prime}\right)} u_{m}^{(s-1)}\right)(y)\right)^{2} d x d y \\
& \leqq \max _{x, y \in \Omega}\left|G^{(2)}(x, y)\right|\left\|\mathbb{G}_{\left(m^{\prime}\right)} u_{m}^{(s-1)}\right\|_{L^{2}(\Omega)}^{4} .
\end{aligned}
$$

We know that

$$
\max _{x, y \in \bar{\Omega}}\left|G^{(2)}(x, y)\right| \leqq \max _{x \in \bar{\Omega}}\left|\int_{\Omega} G(x, z)^{2} d z\right| \leqq C\left(m^{\prime}\right)^{-1 / 2}
$$

Thus, $\mathbb{E}\left(K_{s}\left(u_{m} ; w(m)\right)^{4}\right) \leqq C^{\prime \prime}\left(C^{\prime} m^{\prime}\right)^{-4 s-1 / 2}$. Therefore, we have the following:

Lemma 8. Fix $\varepsilon>0$. Then

$$
\lim _{m \rightarrow \infty} \mathbb{P}_{m}\left((\tilde{m})^{1 / 4}\left(m^{\prime}\right)^{\xi} \sum_{s=1}^{m^{*}}\left(4 \pi \alpha \tilde{m} m^{-1}\right)^{s} K_{s}\left(u_{m} ; w(m)\right) \leqq \varepsilon\right)=1
$$

holds for any $\xi \in[0,1 / 8)$. 
Now we are in a position to state the following:

Proposition 5. Let $\left\{u_{j}\right\}_{j=1}^{\infty}$ be as before. Assume $\gamma>4(\beta-1)$. Then,

$$
\lim _{m \rightarrow \infty} \mathbb{P}_{m}\left((\tilde{m})^{1 / 4}\left(m^{\prime}\right)^{\xi}\left\|\left(\widetilde{\mathbb{H}}_{\left(m^{\prime}\right)}-\widetilde{\mathbb{G}}_{(m)}\right) u_{m}\right\|_{L^{2}(\Omega)} \leqq \varepsilon\right)=1
$$

holds for any $\xi \in[0,1 / 8), \varepsilon>0$.

Proof. We only notice that the last term in (7.1) is negligible in our discussion and we get the desired result. q.e.d.

By essentially the same argument as above we can also prove the following:

Proposition 6. Let $\left\{u_{m}(w(m))\right\}_{m=1}^{\infty}$ be a sequence of $L^{2}(\Omega)$-valued random variables on $\Omega^{\tilde{m}}$ such that $\left\|u_{m}(w(m))\right\|_{L^{2}(\Omega)} \leqq 1$. Assume that $\gamma>4(\beta-1) \geqq 0$. Then,

$$
\lim _{m \rightarrow \infty} \mathbb{P}_{m}\left((\tilde{m})^{1 / 4}\left(m^{\prime}\right)^{\xi}\left\|\left(\mathbb{H}_{\left(m^{\prime}\right)}-\widetilde{\mathbb{G}}_{(m)}\right)\left(u_{m}(w(m))\right)\right\|_{L^{2}(\Omega)} \leqq \varepsilon\right)=1
$$

holds for any $\xi \in[0,1 / 8), \varepsilon>0$.

By a simple calculation we get the following:

Proposition 7. Fix $\beta \in[1,34 / 25)$. Then, there exists $\gamma \in(4(\beta-1), 36 / 25), \kappa^{* *}(\beta)>0$ such that

$$
\lim _{m \rightarrow \infty} \mathbb{P}_{m}\left(m^{\kappa^{* *}(\beta)+2 \gamma-(\beta-1)}\left\|\left(\mathbb{H}_{\left(m^{\prime}\right)}-\widetilde{\mathbb{G}}_{\left(m^{\prime}\right)}\right) \varphi_{j, m}\right\|_{L^{2}(\Omega)} \leqq \varepsilon\right)=1
$$

hold for any $\varepsilon>0$.

A similar result holds when $\varphi_{j, m}$ is replaced by $u_{m}(w(m))$. The statement is denoted by Proposition $7^{\text {bis }}$.

\section{Proof of Theorem 1}

Let $\varphi_{j, m}$ be as before. If $w(m)$ satisfies $(\mathrm{D}-0)_{m}$ and $(\mathrm{D}-\infty)_{m}$, then

$$
\begin{aligned}
\left\|\left(\mathbb{G}_{\left(m^{\prime}\right)}-\tilde{\mu}_{j, m}^{-1}\right) \varphi_{j, m}\right\|_{L^{2}(\omega)} \leqq & \left\|\mathbb{Q}_{\left(m^{\prime}\right)}\right\|_{L^{2}(\omega)}+\left\|\tilde{\mathbb{H}}_{\left(m^{\prime}\right)}\left(\tilde{\chi}_{\omega} \varphi_{j, m}\right)\right\|_{L^{2}(\omega)} \\
& +\left\|\left(\widetilde{\mathbb{G}}_{(m)}-\widetilde{\mathbb{H}}_{\left(m^{\prime}\right)}\right) \varphi_{j, m}\right\|_{L^{2}(\Omega)} .
\end{aligned}
$$

Fix $\beta \in[1,9 / 8)$. Then, we can take $\gamma>4(\beta-1)$ such that Propositions $3,4,7$ hold. Therefore, there exists $\tilde{\kappa}(\beta)>0$ such that

$$
\begin{gathered}
\lim _{m \rightarrow \infty} \mathbb{P}_{m}\left((\mathrm{D}-0)_{m},(\mathrm{D}-\infty)_{m} \text { hold, } m^{\kappa(\beta)+2 \gamma-(\beta-1)}\right. \\
\left.\cdot\left\|\left(\mathbb{G}_{\left(m^{\prime}\right)}-\tilde{\mu}_{j, m}^{-1}\right) \varphi_{j, m}\right\|_{L^{2}(\omega)}=o(1)\right)=1
\end{gathered}
$$

holds. We know from the spectral theory of a self-adjoint compact operator that $\lim _{m \rightarrow \infty} \mathbb{P}_{m}\left((\mathrm{D}-0)_{m},(\mathrm{D}-\infty)_{m}\right.$ hold, there exists at least

of $\mathbb{G}_{\left(m^{\prime}\right)}$ satisfying

$$
m_{\left(\tilde{\mu}_{j, m}\right)} \text {-eigenvalues } \lambda_{q_{s}(w(m))}(w(m)), s=1, \ldots, m_{\left(\tilde{\mu}_{j, m}\right)}
$$

$$
\left.\left|\tilde{\mu}_{j, m}^{-1}-\lambda_{q_{s}(w(m))}(w(m))\right|=o\left(m^{(\beta-1)-2 \gamma-\tilde{\kappa}(\beta)}\right)\right)=1 .
$$

Here $m_{\left(\tilde{\mu}_{j, m}\right)}$ denotes the multiplicity of $\tilde{\mu}_{j, m}$. 
Assume that $w(m)$ satisfies $(\mathrm{D}-0)_{m},(\mathrm{D}-\infty)_{m}$. Let

$$
\lambda_{1}^{*}(w(m)) \geqq \lambda_{2}^{*}(w(m)) \geqq \ldots \downarrow 0
$$

denote the eigenvalues of $\mathbb{G}_{\left(m^{\prime}\right)}$ and $\left\{\varphi_{j}^{*}(w(m))\right\}_{j=1}^{\infty}$ denote a complete orthonormal basis of the eigenfunction of $\mathbb{G}_{\left(m^{\prime}\right)}$ associated with $\lambda_{j}^{*}(w(m))$. Let $\tilde{\varphi}_{j}^{*}(w(m))$ denote the following:

$$
\begin{gathered}
\left(\tilde{\varphi}_{j}^{*}(w(m))\right)(x)=\left(\varphi_{j}^{*}(w(m))\right)(x), \quad x \in \omega, \\
\left(\tilde{\varphi}_{j}^{*}(w(m))\right)(x)=0, \quad x \in \Omega \backslash \bar{\omega} .
\end{gathered}
$$

Then, we see that

$$
\begin{aligned}
& \|\left(\widetilde{\mathbb{G}}_{(m)}-\lambda_{j}^{*}(w(m))\right)\left(\tilde{\varphi}_{j}^{*}(w(m)) \|_{L^{2}(\Omega)}\right. \\
& \quad \leqq \|\left(\tilde{\mathbb{G}}_{(m)}-\widetilde{\mathbb{H}}_{\left(m^{\prime}\right)}\right)\left(\tilde{\varphi}_{j}^{*}(w(m))\left\|_{L^{2}(\Omega)}+\right\| \mathbb{Q}_{\left(m^{\prime}\right)} \|_{L^{2}(\omega)} .\right.
\end{aligned}
$$

Fix $\beta \in[1,9 / 8)$. Then, we can take $\gamma>4(\beta-1)$ such that Propositions $3,7^{\text {bis }}$ hold. Thus,

$$
\begin{aligned}
& \lim _{m \rightarrow \infty} \mathbb{P}_{m}\left((\mathrm{D}-0)_{m}(\mathrm{D}-\infty)_{m} \text { hold, there exist } \kappa^{*}(\beta)>0\right. \\
& \text { and at least } m_{\lambda_{j}^{*}(w(m))^{-}} \text {-eigenvalues } \\
& \left(\tilde{\mu}_{r_{t}(w(m)), m}\right)^{-1}, t=1, \ldots, m_{\lambda_{j}^{*}(w(m))} \text { of } \widetilde{\mathbb{G}}_{(m)} \\
& \text { satisfying }\left|\lambda_{j}^{*}(w(m))-\left(\mu_{r_{t}(w(m)), m}\right)^{-1}\right| \\
& \left.\quad=o\left(m^{(\beta-1)-2 \gamma-\kappa^{*}(\beta)}\right)\right)=1 .
\end{aligned}
$$

In summing up these facts we get the following:

Proposition 8. Fix $\beta \in[1,9 / 8)$. Then, we can take $\gamma>4(\beta-1)$ such that

$$
\begin{gathered}
\lim _{m \rightarrow \infty} \mathbb{P}_{m}\left((\mathrm{D}-0)_{m},(\mathrm{D}-\infty)_{m} \text { hold, there exists } \kappa(\beta)>0\right. \\
\text { such that } \left.\left|\lambda_{j}^{*}(w(m))^{-1}-\tilde{\mu}_{j, m}\right|=0\left(m^{(\beta-1)-\kappa(\beta)}\right)\right)=1 .
\end{gathered}
$$

Here we used the fact that $\tilde{\mu}_{j, m}$ can be written as

$$
\tilde{\mu}_{j, m}=m^{\gamma}+4 \pi \alpha m^{\beta-1} v_{m, j},
$$

where

$$
v_{m, j} \in(\min V(x) / 2, \max V(x) / 2)
$$

for large $m$. Fix $j$. We will show that

$$
\lambda_{j}^{*}(w(m))^{-1}=\mu_{j}(\alpha / m ; w(m))+m^{\prime}
$$

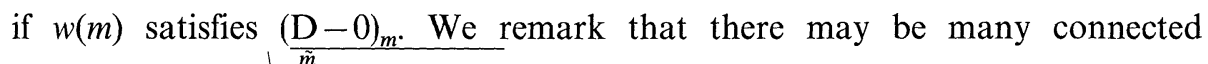
components of $\Omega \bigcup_{i=1}^{m} B\left(\alpha / m ; w_{i}^{(m)}\right)$. Thus, $\mu_{j}(\alpha / m ; w(m))$ may come from $\omega_{s}(w(m))$ for $s \geqq 2$. We know by the properties of eigenvalues of $-\Delta+m^{\prime}$ in $\omega_{s}(w(m)), s \geqq 2$ that they are at least of order $m^{2}(\log m)^{-2}$ as $m \rightarrow \infty$. Thus, we get (7.12). In summing up the above facts we get Theorem 1 . 


\section{Short Discussion}

In Ozawa [5], the author used Kac's theorem (=Theorem A in this note with $\tilde{\delta}=0, \beta=1)$ to prove Theorem A with $\tilde{\delta}>0, \beta=1$. By the method developed in this paper, we get Theorem A without using the theory of Brownian motion. The author hopes here that we can get Theorem 1 by purely probabilistic methods.

The author should remark that there is a small modifiable mistake in the proof of Ozawa [5, Proposition 4]. The formula (3.9) in [5] is not correct, however, Proposition 4 in [5] still remains correct. The proof can be obtained by using a formula and method like (7.1) of this paper.

Acknowledgement. The author expresses his sincere thanks to Professors G. C. Papanicolaou (Courant Institute) and N. Ikeda (Osaka University) for their invaluable support to the author.

\section{References}

1. Bensoussan, A., Lions, J.L., Papanicolaou, G.C.: Asymptotic methods in periodic structures. Amsterdam: North-Holland 1978

2. Huruslov, E.Ja., Marchenko, V.A.: Boundary value problems in regions with fine-grained boundaries (in Russian). Kiev 1974

3. Kac, M.: Probabilistic methods in some problems of scattering theory. Rocky Mountain J. Math. 4, 555-538 (1974)

4. Lions, J.L.: Some methods in mathematical analysis of systems and their control. New York: Gordon and Breach 1981

5. Ozawa, S.: On an elaboration of M. Kac's theorem concerning eigenvalues of the Laplacian in a region with random distributed small obstacles. Commun. Math. Phys. 91, 473-487 (1983)

6. Ozawa, S.: Spectra of domains with small spherical Neumann boundary. J. Fac. Sci. Univ. Tokyo, Sect. IA 30, 259-271 (1983)

7. Papanicolaou, G.C., Varadhan, S.R.S.: Diffusion in region with many small holes. In: Lecture Notes in Control and Information, Vol.75, pp. 190-206. Berlin, Heidelberg, New York: Springer 1980

8. Papanicolaou, G.C., Varadhan, S.R.S.: Boundary value problems with rapidly oscillating random coefficients. Coll. Math. Soc. János Bolyai 27, Random fields, Vol. II, pp. 835-873, Fritz, J., Lebowitz, J.L., Szász, D. (eds.). Amsterdam: North-Holland 1981

9. Rauch, J., Taylor, M.: Potential and scattering theory on wildly perturbed domains. J. Funct. Anal. 18, 27-59 (1975)

10. Simon, B.: Functional integration and quantum physics. New York: Academic Press 1979

Communicated by T. Spencer

Received October 5, 1983; in revised form April 16, 1984 
